Proceedings

\title{
Microfluidic Portable Device for Pathogens 'Rapid SERS Detection ${ }^{+}$
}

\author{
Nicoleta Elena Dina 1,*, Alia Colniță ${ }^{1}$, Daniel Marconi ${ }^{1}$ and Ana Maria Raluca Gherman ${ }^{1,2}$ \\ 1 Department of Molecular and Biomolecular Physics, National Institute for Research and Development of \\ Isotopic and Molecular Technologies, 400293 Cluj-Napoca, Romania; \\ 2 Faculty of Physics, Babes-Bolyai University, 400084 Cluj-Napoca, Romania \\ * Correspondence: nicoleta.dina@itim-cj.ro \\ + Presented at the 1st International Electronic Conference on Biosensors, 2-17 November 2020; Available \\ online: https://iecb2020.sciforum.net/.
}

Received: date; Accepted: date; Published: date

\begin{abstract}
So far, in some of our previous works we have managed to rapidly (within minutes) identify and discriminate pathogens by using surface-enhanced Raman scattering (SERS) spectroscopy with a single cell sensitivity. Having a more user friendly and robust system, which could be used not only by experts, would be the next step. In order to meet our goal, we developed an experimental setup, including an in-house built microfluidic device followed by the optimization of SERS detection of common bacterial pathogens by using the developed device. The main components of the system are: a microfluidic flow-cell coupled to a syringe pump mediated-flow system, and a portable Raman spectrometer for detecting the bacteria immobilized in the flow cell. Inside the microfluidic channel of the flow cell, a silver spot was generated under laser irradiation for further use as SERS active substrate for detection. The silver spot can be washed and reused for a different pathogen from one experiment to another. No specific capturing receptors are used. The total analysis time was reduced to less than $15 \mathrm{~min}$. Considering the fit-for-purposes experimental parameters for detection and its easy-to-use dedicated software, this portable microfluidic device has been tested in our lab and is ready to be transferred in the research/clinical premises for further use.
\end{abstract}

Keywords: biosensing; SERS on bacteria; microfluidic device

\section{Introduction}

PDMS is one of the most actively developed polymers for microfluidics designing. The fabrication of channel systems in PDMS-poly(dimethylsiloxane) is facilitated by the fact that it can be cast using a suitable mold with sub- $0.1 \mu \mathrm{m}$ fidelity. Being also more than a structural material it allows the fabrication of devices with useful functionality due to its chemical and physical properties: elasticity, optical transparency, low water permeability, low electrical conductivity, and the possibility of its patterning by lithographic techniques [1].

Usually, the pathogen's detection is based on immobilization by using specific antigen, antibodies, labelling molecule etc. Moreover, several approaches of capturing/concentration/filtration are used for having a significant biomass to detect accurately. For instance, the on-line SERS detection and accurate identification of suspended bacteria with a detection capability down to a single bacterium has been realized by the nanoaggregate-embedded beads (NAEBs)-dielectrophoresis (DEP)-Raman spectroscopy biosensing strategy [2].

In a study from 2013, Chrimes et al. [3] reports the fabrication of microfluidic channels with a size of $150 \times 70 \mu \mathrm{m}^{2}$ and a length of $12 \mathrm{~mm}$, made of PDMS on quartz substrate, the latter being chosen due to the very weak Raman signal and interference with the SERS signal from the analyte (yeast Saccharomyces cerevisiae). The microfluidic system was connected to an injection pump to facilitate the 
transport of the yeasts through the microfluidic channels. To perform the measurement, the yeasts coated with Ag nanoparticles (AgNPs) were manipulated and immobilized inside the microchannel by using DEP force field. Another study [4] reports the development of a microfluidic platform for the detection of several pathogenic bacteria using AgNPs as a SERS substrate. In this case, the microfluidic channels were also made in PDMS on glass substrate by means of photolithography method.

We herein propose a practical detection approach as an alternative to conventional methods, by developing an experimental setup based on the principle of in situ preparation of silver SERS-active substrate, in a Plexiglas microfluidic detection cell, by using the portable Raman spectrometer as detection tool.

\section{Materials and Methods}

For bacterial cells activation, aliquot with E. coli TOP10, P. aeruginosa, S. aureus and respectively E. faecalis-stored at $-80^{\circ} \mathrm{C}$ for long term storage was previously partially thawed at room temperature. After thawing, $1 \mu \mathrm{L}$ of bacteria was transferred using a sterile plastic loop into a new tube containing $5 \mathrm{~mL}$ of liquid Luria Broth (LB) media. The LB medium was previously sterilized by autoclaving at $121^{\circ} \mathrm{C}$ for $5 \mathrm{~min}$. The tube containing media and the inoculum was vortexed for $5 \mathrm{~s}$ (max. speed) and then incubated overnight at $37^{\circ} \mathrm{C}$. To obtain pure colonies, $10 \mu \mathrm{L}$ from overnight culture was then subjected to serial dilution technique. After inoculation of LB medium with the desired dilution, the Petri dishes were incubated $15 \mathrm{~min}$ at room temperature and after were transferred to the incubator at $37^{\circ} \mathrm{C}$ overnight (at least $20 \mathrm{~h}$ ).

\section{Standard SERS samples preparation.}

After incubation, we inspected the Petri dishes and a single colony of bacteria was transferred into $5 \mathrm{~mL}$ of sterile LB Broth and grown overnight at $37^{\circ} \mathrm{C}$. After growing, $4 \mathrm{~mL}$ of $E$. faecalis culture were centrifuged at $3000 \times g$ for $2 \mathrm{~min}$, in two steps ( $2 \mathrm{~mL}$ of bacterial culture for each step), the supernatant was discarded and the remained pellet was resuspended (washed) in $2 \mathrm{~mL}$ of sterile saline solution and gentle vortexed. This washing protocol was repeated three times for a thorough discard of the growth components. The working culture was obtained through resuspension of the obtained pellet (after three steps of washing) in $200 \mu \mathrm{L}$ of sterile saline solution. The working culture was stored at $4{ }^{\circ} \mathrm{C}$ until further analysis. If the bacterial suspension was too concentrated, further dilutions were carried out with the same saline solution.

SERS spectra acquisition. The Raman system used to perform the SERS experiments consisted in a BW-TEK portable i-Raman spectrometer equipped with a $532 \mathrm{~nm}$ laser line and a total power source of $50 \mathrm{~mW}$, connected through an optical fiber to a BW-TEK optical microscope equipped with $20 \times$ objective. A syringe with $3 \mathrm{~mL}$ of the bacterial biomass was attached to the two-channel injection pump (NE-4000 Programmable 2 Channel Syringe Pump, NewEra Pump Systems Inc.) used in the microfluidic SERS-based detection setup.

\section{Results}

\subsection{Experimental Setup}

Figure 1 shows the microfluidic cell placed on the table of a Raman microscope, which is connected to both the portable Raman spectrometer equipped with the laser line with wavelength of $532 \mathrm{~nm}$ (through fiber optics), as well as the computer equipped with dedicated software for precise laser control and accurate recording of SERS spectra. The two inlets are connected to the injection syringe pump. The detection of microorganisms takes place inside a microfluidic channel with an approximate width and height of $500 \mu \mathrm{m}$, carved by using a numerically controlled mechanical milling machine with the help of Microorganisms are SERS detected inside the microfluidic cell, avoiding thus contamination. After passing through the microfluidic channel and the bacteria detection process is complete, the biowaste is disposed through outlet in a special container. 


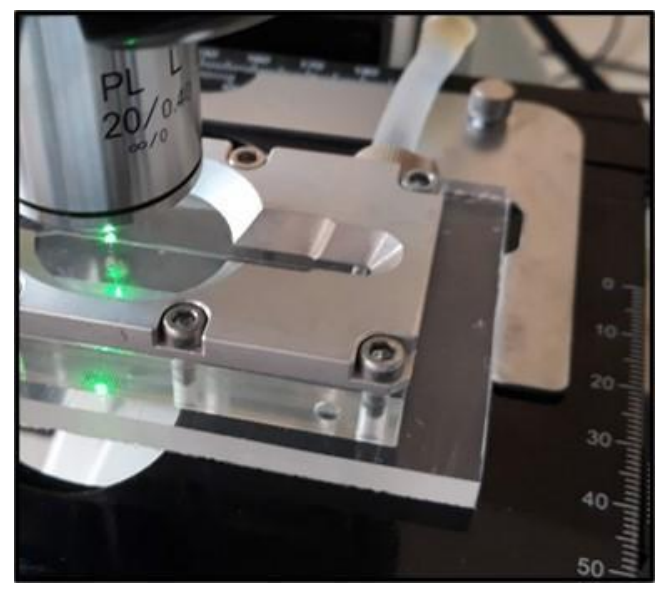

Figure 1. Lateral view showing the microchannel of the microfluidic cell. The $532 \mathrm{~nm}$ laser line is also visible during measurement.

\subsection{Synthesis of the Silver Spot Used for SERS-Based Detection of Bacteria}

In the preparation of the SERS amplifier substrate we used the recipe published by K. Herman et al. [5], with some modifications: while the spot was obtained by the authors of the article inside a glass capillary tube by injecting a mixture of the two components, in our case, we used an injection pump which allows the simultaneous injection, with the same flow rate, of silver nitrate $\left(\mathrm{AgNO}_{3}\right)$ and sodium $(\mathrm{Na})$ citrate inside the channel in the microfluidic cell.

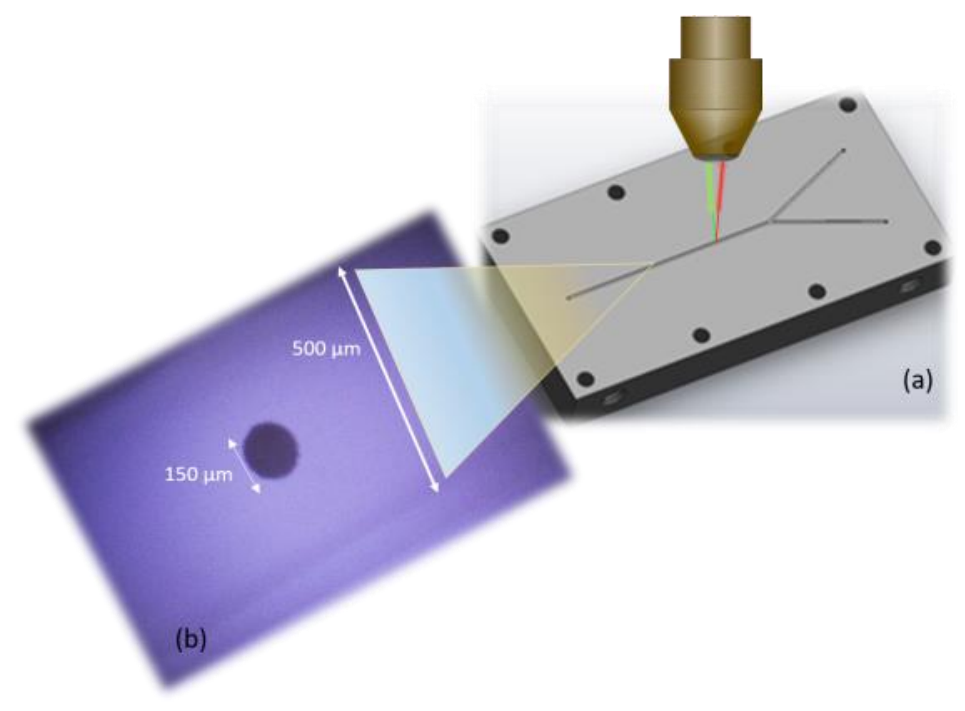

Figure 2. (a) Schematic image of the inside of the microfluidic cell ( $Y$ junction) showing also the silver spot generation process; (b) Microscopic view (20×) of the silver spot generated in the microchannel during laser irradiation.

\subsection{The Detection Process of Microorganisms by Using SERS in the Microfluidic Cell}

The detection process of microorganisms by using SERS takes place inside a microfluidic cell and uses as enhancement substrate an Ag spot synthesized in situ, under $532 \mathrm{~nm}$ laser irradiation. In short, the detection process comprises several steps:

- Setting the flow parameters in the double syringes injection pump: flow rate of $0.25 \mathrm{~mL} / \mathrm{min}$; syringe diameter: $12.97 \mathrm{~mm}$; volume discharged: $5 \mathrm{~mL}$. 
- Setting the laser power to $5 \mathrm{~mW}$ for the portable i-Raman BW-TEK spectrometer connected to the microscope by an optical fiber.

- Concomitantly starting the laser and the injection pump with syringes: the microfluidic channel is filled with the two components; local irradiation and heating by using the $532 \mathrm{~nm}$ laser Ag reduces to $\mathrm{Ag}^{0}$ and an $\mathrm{Ag}$ spot with plasmonic properties is created.

- After the completion of the Ag spot generation process, the SERS substrate is tested by recording the bacteria specific spectra.

- Recording SERS spectra and optimizing detection by testing the acquisition time and laser power appropriate in order to maintain the viability of the samples.

Using a live CCD camera, the irradiated bacterial field was visualized in real time, prior and during the spectral measurements. Before the SERS measurement was made, the laser spot hit isolated bacteria cells, with dimensions similar as the laser spot (with a total area of $2 \mu \mathrm{m}^{2}$ ).

\section{Discussion}

We consider that due to the fact that the Ag spot is formed on a solid surface (inside the microfluidic channel) and composed of Ag nanoclusters, the injected bacteria are adsorbed on the SERS substrate, due to the higher affinity of the cell wall components for the Ag nanoclusters than the existing citrate ions. Figure 3 shows the SERS spectra of bacteria detected inside the microfluidic channel for 4 bacterial species. Both Gram-positive and Gram-negative species' spectral fingerprints were successfully recorded. At least 3 of the SERS marker bands were detected for each species (725 $\mathrm{cm}^{-1}, 1089 \mathrm{~cm}^{-1}$ and respectively $1316 \mathrm{~cm}^{-1}$ ), with slight Raman shifting from one species to another. These bands are assigned to living bacteria, since antibiotic stress or lysis is known to affect significantly [6] the strong $725 \mathrm{~cm}^{-1}$ and $1316 \mathrm{~cm}^{-1}$ bands attributed to adenine and guanine respectively, specific to SERS bacterial fingerprint [7].

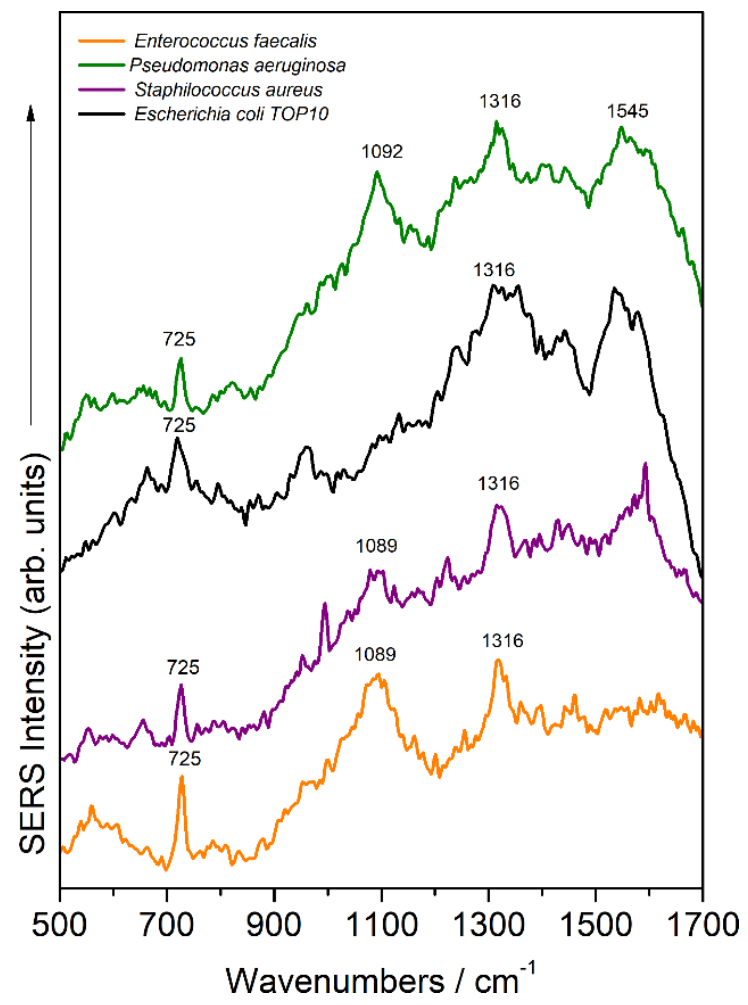

Figure 3. SERS spectra of bacteria detected inside the microfluidic channel by using the Ag spot synthesized in situ as enhancement substrate. 
It is known that sample preparation for the SERS detection of bacteria is quite inconsistent referring to colloids as SERS active substrates. The NPs can be either coated on the outside of the bacterial cell wall or directed to the interior of the bacterial cells. Whereas the first preparation results in spectral information mainly derived from cell wall components and the second one contains additional cytoplasm information. By employing the herein described in situ preparation of silver SERS-active substrate, the spectral fingerprint obtained is from the cell wall of bacteria.

Conventional synthesis of colloidal suspension by using some reducing agents may result in the presence of extra ions originating from the reaction process [8]. The instability of the sols has been one of our concerns and the presence of certain ions in the sols might be one reason for the unstable behavior of the sols. By using this preparation method, the presence of any byproducts and the instability of the nanoparticles are highly reduced. Additionally, spectral fluctuations deriving from the drying process are avoided and the spectral contributions recorded possess high specificity, arising for modes of flavin derivates prone to the SERS effect [9].

Therefore, we believe that this is a promising technique for further relevant applications, wherever reliable bacteria validation is required.

\section{Patents}

A national patent registered at OSIM with nr. A00976/28.11.2018 resulting from the work reported in this manuscript.

Author Contributions: Conceptualization, N.E.D. and D.M.; methodology, A.C.; software and validation, A.M.R.G. All authors have read and agreed to the published version of the manuscript.

Funding: This research was funded by Romanian Ministry of Education (RME), CCCDI-UEFISCDI: grant numbers PN-III-P2-2.1-PED-2016-0983 (microfluidic device fabrication) and PN-III-P1-1.1-TE-2019-0910 (microfluidic device is still been used at the moment for SERS detection of bacteria).

Acknowledgments: The authors gratefully appreciate the help of Tiberiu Szöke-Nagy from National Institute for Research and Development of Isotopic and Molecular Technologies, Molecular and Biomolecular Physics Department for providing the bacterial biomass in standardized samples.

Conflicts of Interest: The authors declare no conflict of interest.

\section{References}

1. Ng, J.M.; Gitlin, I.; Stroock, A.D.; Whitesides, G.M. Components for integrated poly(dimethylsiloxane) microfluidic systems. Electrophoresis 2002, 23, 3461-3473, doi:10.1002/1522-2683(200210)23:203.0.CO;2-8.

2. Lin, H.-Y.; Huang, C.-H.; Hsieh, W.-H.; Liu, L.-H.; Lin, Y.-C.; Chu, C.-C.; Wang, S.-T.; Kuo, I.-T.; Chau, L.K.; Yang, C.-Y. On-line SERS Detection of Single Bacterium Using Novel SERS Nanoprobes and A Microfluidic Dielectrophoresis Device. Small 2014, 10, 4700-4710, doi:10.1002/smll.201401526.

3. Chrimes, A.F.; Khoshmanesh, K.; Tang, S.-Y.; Wood, B.R.; Stoddart, P.R.; Collins, S.S.; Mitchell, A.; Kalantar-Zadeh, K. In situ SERS probing of nano-silver coated individual yeast cells. Biosens. Bioelectron. 2013, 49, 536-541, doi:10.1016/j.bios.2013.05.053.

4. Mungroo, N.A.; Oliveira, G.; Neethirajan, S. SERS based point-of-care detection of food-borne pathogens. Microchim. Acta 2015, 183, 697-707, doi:10.1007/s00604-015-1698-y.

5. Herman, K.; Mircescu, N.E.; Szabo, L.; Leopold, L.F.; Chiş, V.; Leopold, N. In situ Silver Spot Preparation and on-Plate Surface-Enhanced Raman Scattering Detection in Thin Layer Chromatography Separation. J. Appl. Spectrosc. 2013, 80, 311-314, doi:10.1007/s10812-013-9765-9.

6. Lemma, T.; Saliniemi, A.; Hynninen, V.; Hytönen, V.P.; Toppari, J.J. SERS detection of cell surface and intracellular components of microorganisms using nano-aggregated Ag substrate. Vib. Spectrosc. 2016, 83, 36-45, doi:10.1016/j.vibspec.2016.01.006.

7. Kang, J.W.; So, P.T.C.; Dasari, R.R.; Lim, D.-K. High Resolution Live Cell Raman Imaging Using Subcellular Organelle-Targeting SERS-Sensitive Gold Nanoparticles with Highly Narrow Intra-Nanogap. Nano Lett. 2015, 15, 1766-1772, doi:10.1021/n1504444w. 
8. Zhang, Y.; Wang, F.; Yin, H.; Hong, M. Nonuniform Distribution of Capping Ligands Promoting Aggregation of Silver Nanoparticles for Use as a Substrate for SERS. Adv. Nanoparticles 2013, 2, 104-111, doi:10.4236/anp.2013.22018.

9. Efrima, S.; Zeiri, L. Understanding SERS of bacteria. J. Raman Spectrosc. 2009, 40, 277-288, doi:10.1002/jrs.2121.

Publisher's Note: MDPI stays neutral with regard to jurisdictional claims in published maps and institutional affiliations.

(C) 2020 by the authors. Submitted for possible open access publication under the terms and conditions of the Creative Commons Attribution (CC BY) license (http://creativecommons.org/licenses/by/4.0/). 